\title{
Use and Meaning of "Goals of Care" in the Healthcare Literature: a Systematic Review and Qualitative Discourse Analysis
}

\author{
Katharine Secunda, $M D^{7,2}$, M Jeanne Wirpsa, MA, BCC $C^{3}$, Kathy J Neely, MD, MA ${ }^{7,4}$, \\ Eytan Szmuilowicz, MD ${ }^{1,4}$, Gordon J Wood, MD ${ }^{1,4}$, Ellen Panozzo, LCSW', \\ Joan McGrath, RN ${ }^{3}$, Anne Levenson, MD ${ }^{1,2}$, Jonna Peterson, MLIS ${ }^{1,5}$, \\ Elisa J Gordon, $\mathrm{PhD}, \mathrm{MPH}^{1,6,7,8}$, and Jacqueline $M$ Kruser, $M D, M S^{1,2,7}$
}

${ }^{1}$ Northwestern University Feinberg School of Medicine, Chicago, IL, USA; ${ }^{2}$ Department of Medicine, Division of Pulmonary and Critical Care, Northwestern University Feinberg School of Medicine, Chicago, USA; ${ }^{3}$ Northwestern Memorial Hospital, Chicago, IL, USA; ${ }^{4}$ Department of Medicine, Division of Hospital Medicine, Northwestern University Feinberg School of Medicine, Chicago, USA; ${ }^{5}$ Galter Health Sciences Library and Learning Center, Northwestern University Feinberg School of Medicine, Chicago, USA; ${ }^{\circ}$ Department of Surgery, Division of Transplantation, Northwestern University Feinberg School of Medicine, Chicago, USA; ${ }^{7}$ Center for Health Services and Outcomes Research, Northwestern University Feinberg School of Medicine, Chicago, USA; ${ }^{8}$ Center for Bioethics and Medical Humanities, Northwestern University Feinberg School of Medicine, Chicago, USA.

BACKGROUND: The specific phrase "goals of care" (GOC) is pervasive in the discourse about serious illness care. Yet, the meaning of this phrase is ambiguous. We sought to characterize the use and meaning of the phrase GOC within the healthcare literature to improve communication among patients, families, clinicians, and researchers. METHODS: A systematic review of the English language healthcare literature indexed in MEDLINE/PubMed, Cumulative Index to Nursing and Allied Health Literature (CINAHL), and Scopus was performed in October of 2018. We searched for all publications with the exact phrase "goals of care" within the title or abstract; no limitations on publication date or format were applied; conference abstracts were excluded. We used qualitative, discourse analysis to identify key themes and generate an operational definition and conceptual model of GOC.

RESULTS: A total of 214 texts were included in the final analysis. Use of GOC increased over time with $87 \%$ of included texts published in the last decade (2009-2018). An operational definition emerged from consensus within the published literature: the overarching aims of medical care for a patient that are informed by patients' underlying values and priorities, established within the existing clinical context, and used to guide decisions about the use of or limitation(s) on specific medical interventions. Application of the GOC concept was described as important to the care of patients with serious illness, in order to (1) promote patient autonomy and patient-centered care, (2) avoid unwanted care and identify valued care, and (3) provide psychological and emotional support for patients and their families.

Prior Presentations A portion of the findings reported in this manuscript were presented at the American Thoracic Society International Conference in Dallas, TX, in May 2019.

Electronic supplementary material The online version of this article (https://doi.org/10.1007/s11606-019-05446-0) contains supplementary material, which is available to authorized users.

Received August 7, 2019

Accepted September 26, 2019

Published online October 21, 2019
DISCUSSION: The use of the phrase "goals of care" within the healthcare literature is increasingly common. We identified a consensus, operational definition that can facilitate communication about serious illness among patients, families, and clinicians and provide a framework for researchers developing interventions to improve goalconcordant care.

KEY WORDS: palliative care; terminology; terminal care; systematic review; discourse analysis.

J Gen Intern Med 35(5): 1559-66

DOI: $10.1007 / \mathrm{s} 11606-019-05446-0$

(c) Society of General Internal Medicine 2019

\section{INTRODUCTION}

The specific phrase "goals of care" (GOC) is used pervasively by clinicians, researchers, and health policymakers who aim to provide the best possible care for patients with serious illnesses. Despite widespread use, the meaning of this phrase is ambiguous and lacks a consensus or operational definition. ${ }^{1}$ In prior work, we found the phrase GOC is ubiquitous in clinician notes within the electronic health records (EHR) of critically ill patients admitted to an intensive care unit. ${ }^{2}$ In the EHR, GOC is used by clinicians to represent communication about poor prognosis, to describe conflict among clinicians, patients, and families, and to provide a rationale for specific treatment limitations. However, the phrase was rarely used to convey specific patient or family goals or values.

There is well-recognized ambiguity around many common phrases that refer to the care of patients with life-limiting illness, including "seriously ill," "comfort care," "supportive care," and the phrase "palliative care" itself. ${ }^{3-5}$ Using euphemisms and vague language in communication about serious 
illness, death, and dying can have important consequences for patient care, including misunderstanding and miscommunication among patients, families, and interprofessional clinical teams. ${ }^{6}$ Lack of precision and inconsistency in definitions also creates a barrier for researchers who aim to improve patientcentered decision-making, palliative care, and end-of-life care. $^{7}$ The National Institutes of Health and the American Society for Clinical Oncology have both declared the need to clarify palliative care terminology to improve the systematic investigation of end-of-life care and to advance the science of palliative care. ${ }^{4,8}$

The objectives of this study were to characterize the use and meaning of the phrase GOC within the healthcare literature pertaining to patients with serious illness, and to identify an operational definition based on consensus within the literature that can be used to facilitate accurate and meaningful communication among patients, their families, clinicians, and researchers.

\section{METHODS}

\section{Healthcare Literature Systematic Review}

In October 2018, a medical librarian (JP) conducted a search for the exact phrase "goals of care" in the title and abstract field in each of the following databases: MEDLINE via PubMed, Cumulative Index to Nursing and Allied Health Literature (CINAHL), and Scopus. Searches were limited to English language texts; conference proceedings were excluded. Given our objective to characterize the use and meaning of GOC within the healthcare literature, we did not restrict the search results by date of publication, publication format, or study design. Texts with trivial use of the phrase GOC and texts that did not relate to patients with serious, lifethreatening, or life-limiting illness were excluded. Two investigators (KS and JMK) screened all texts for inclusion using the systematic review processing software Rayyan (https://rayyan.qcri.org); all disagreements were adjudicated by a third reviewer (AL).

Our search strategy yielded 1645 texts for initial review (Fig. 1); 290 were potentially eligible after title/abstract screening. After exclusion of 71 texts that did not meet inclusion criteria, 4 duplicate texts, and 1 text that was unavailable for full-text review, a total of 214 texts were included in the final sample (see Appendix in Supplemental Materials for full reference list).

\section{Data Extraction and Qualitative Analysis}

We conducted a qualitative discourse analysis ${ }^{9,10}$ to evaluate use and meaning of the phrase GOC within the healthcare literature. Discourse analysis refers to a method that evaluates the application of language and text within its natural context. ${ }^{11}$ Given the phrase GOC is rarely accompanied by an

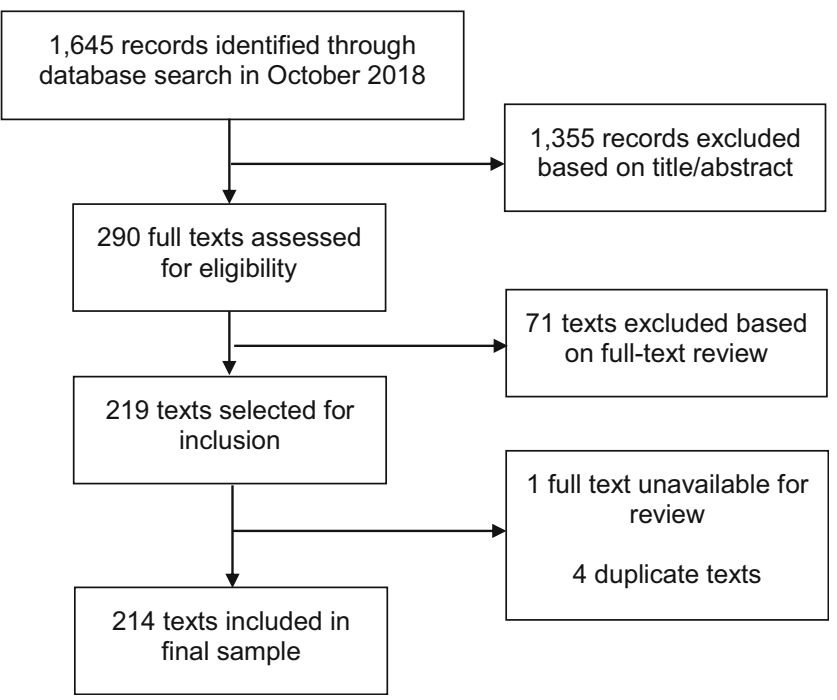

Figure 1 Search strategy and results. Selection of texts after application of inclusion and exclusion criteria is shown.

explicit definition in the healthcare literature, we employed this method to uncover tacit, fundamental themes related to GOC.

In the initial phase of analysis, two investigators (KS and JMK) independently reviewed the title, abstract, manuscript text, tables, and figures of each text to identify every instance of the phrase GOC. The two investigators independently coded each instance of GOC, using an inductive approach to coding $^{12,13}$ to describe characteristics of the use and the meaning of the phrase. The two investigators met weekly to review coded texts and establish a consensus code for each instance of GOC. The individual codes were collated into a coding taxonomy, which was iteratively revised throughout the coding process.

The initial qualitative coding began with the oldest text and proceeded in chronological order. Initial theoretical saturation was reached after coding of the first 56 texts (through the year 2012). To ensure that we did not omit important or novel themes from more recent publications, we used purposive sampling ${ }^{14}$ to select additional texts for full qualitative coding. Two investigators independently reviewed all remaining titles and determined eligibility for inclusion. Criteria for inclusion were (1) previously underrepresented patient populations (e.g., heart failure, surgical patients) and settings (e.g., medical emergency team calls); (2) novel potential themes (e.g., the impact of clinician bias on GOC; documentation of GOC in the health record); or (3) texts that explicitly described reporting a conceptual model of GOC. After independent review, the two investigators met to compare selected titles and reconciled all discrepancies by consensus; 34 additional texts were included in the final purposive sample. We then coded all 34 texts in chronological order beyond theoretical saturation to confirm that no new themes emerged at the conclusion of coding. In total, we qualitatively coded the full-text manuscript of 90 of the 214 texts. 
We conducted axial coding with an interprofessional (5 physicians, 1 nurse, 1 social worker, 1 chaplain) group of investigators with diverse clinical expertise (critical care, palliative care, clinical ethics) to generate themes from and establish relationships among existing codes. ${ }^{13,15}$ During axial coding, we generated an operational definition of GOC based on consensus themes that were identified during discourse analysis. We held weekly, one-hour meetings of at least five investigators to review codes, themes, and definition components until consensus was reached on the key thematic results.

\section{RESULTS}

\section{Use of GOC}

The earliest included text was published in 1987, and the use of GOC within in the healthcare literature increased markedly over time (Fig. 2). Most included texts (87\%) were published in the last decade (2009 to 2018). Included texts were published in 122 unique journals and 5 book chapters. Texts were most frequently published in palliative care journals $(28.0 \%)$, followed by internal medicine/medicine (16.8\%), oncology $(8.4 \%)$, pulmonary/critical care $(7.5 \%)$, nursing $(5.1 \%)$, cardiology $(3.7 \%)$, health services $(3.7 \%)$, geriatrics $(3.3 \%)$, emergency medicine $(1.9 \%)$, general surgery $(1.9 \%)$, and ethics $(1.4 \%)$. Four additional journal categories combined (obstetrics, primary healthcare, pediatrics, and education) accounted for the remaining $3.9 \%$ of texts. Of all included texts, $12.1 \%$ were published in journals not indexed for MEDLINE and $2.3 \%$ were book chapters.

The use of GOC within the healthcare literature referred, primarily, to communication with or about patients with serious illness in the following three categories: (1) patients with diseases typically considered to have a poor prognosis (i.e., advanced cancer or dementia); (2) patients who had reached a phase of any disease for which cure was not possible or death was imminent; and (3) patients who were receiving care within a specific healthcare setting (e.g., nursing home, emergency department, or intensive care unit) where incurable, serious, life-limiting, and life-threatening illnesses are common. Authors also described the healthcare system itself as having a default or implicit GOC to cure disease and prolong life. For example, one author noted that "The implicit goal of health care is usually cure or survival [...], so in many clinical areas, GOC are 'reasonably taken for granted' and not explicitly stated." When GOC was applied to patient populations in the healthcare literature, it typically referred to communication with or about patients for whom the implicit system GOC was no longer possible or likely.

\section{Definition of GOC}

We generated an operational definition and conceptual model of GOC (Fig. 3) based on consensus, key themes within the healthcare literature. GOC are operationally defined as the overarching aims of medical care for a patient that are informed by patients' underlying values and priorities, established within the existing clinical context, and used to guide decisions about the use of or limitation on specific medical interventions. Table 1 displays representative quotations that illustrate the four key themes of this consensus definition and the relationships among the themes.

The first key theme (overarching aims of medical care) is exemplified by authors' frequent use of words that convey the broad scope of the phrase: "overarching," "big picture," "global," "basic orientation," "general," and "overall." One text described the scope of GOC as "more specific than overarching values statements, yet more general than interventions."16 In addition, authors stated that GOC must encompass an "aim," "purpose," "direction," or "telos" towards which medical care is directed.

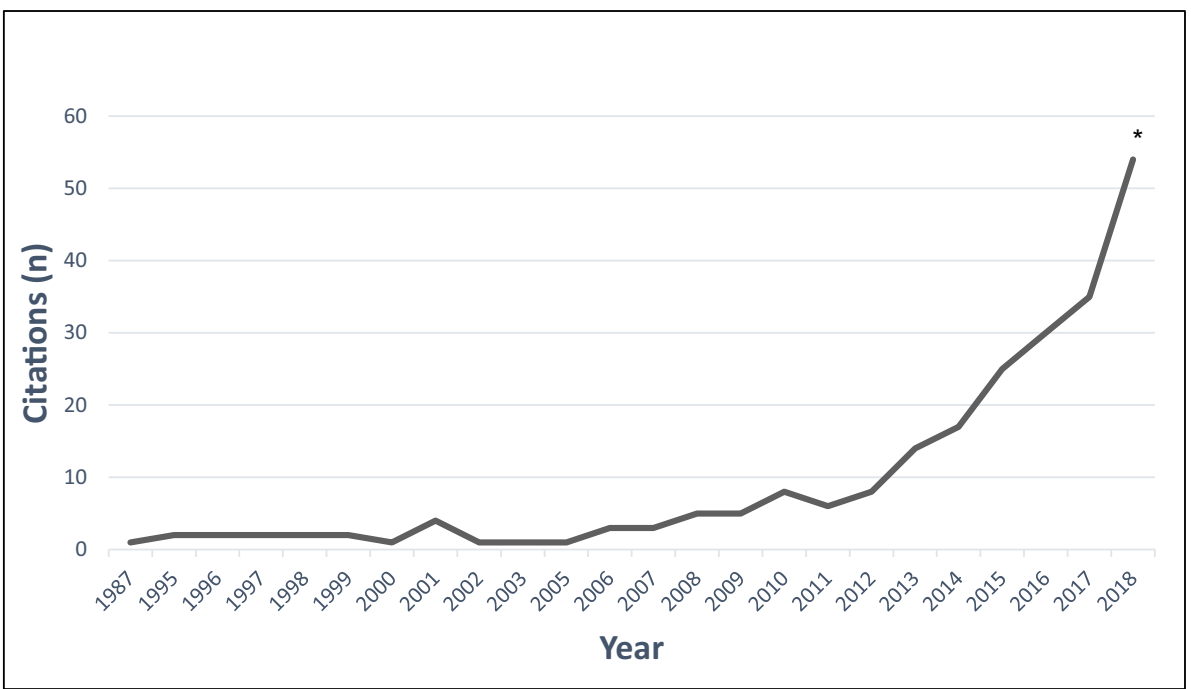

Figure 2 Texts published with a primary focus on "goals of care" have increased over time. The number of "goals of care" citations per year from 1987 to 2018 is shown. *Projected total citations based on the first eight months of 2018. 


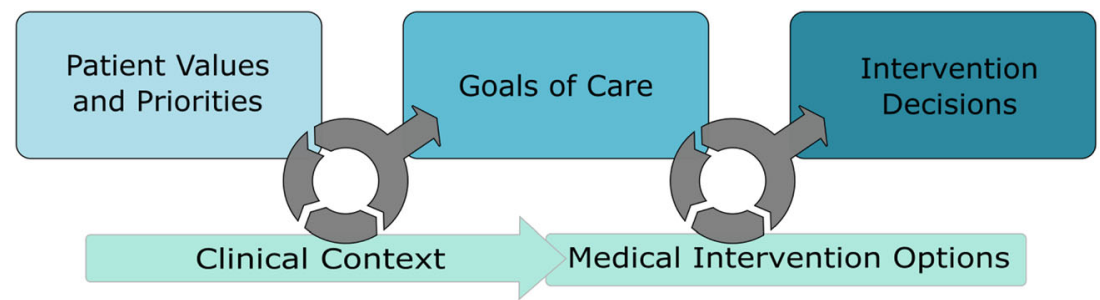

Figure 3 Conceptual model that illustrates the consensus, operational definition of the phrase "goals of care." Goals of care can be defined as the overarching aims of medical care for a patient that are informed by patients' underlying values and priorities, established within the existing clinical context, and used to guide decisions about the use of or limitation on specific medical interventions.

Table 1 The Key Themes That Comprise the Operational Definition of "Goals of Care"

\begin{tabular}{ll}
\hline \hline Key theme & Exemplary quotes \\
\hline Overarching aims & "Goals of care point to a general direction
\end{tabular}
of what is hoped to be achieved through the provision of care."

"[...] the goal speaks to the overarching aim of care. The goal of care indicates the destination and the care plan, how to get there."

Patient values and priorities

Clinical context

Medical intervention decisions

Relationship among key GOC themes
The second key theme (patient values and priorities) requires that GOC be developed "in the context of the patient's values" or "to reflect patient values," including the patient's prioritization or ranking of the relative value of possible outcomes. The third key theme (clinical context) emphasizes the importance of formulating GOC within the bounds of a patient's current clinical context and illness: "an accurate illness understanding is required to meaningfully consider and prioritize $\mathrm{GoC}[\mathrm{sic}] .{ }^{\prime 17} \mathrm{Au}-$ thors used terms such as "appropriate," "attainable," "realistic," "reasonable," and "possible" to convey that GOC are often limited by a patient's underlying illness or prognosis.

The fourth key theme (medical intervention decisions) explicitly differentiates GOC from individual or specific medical intervention decisions but describes GOC as a construct to help guide these decisions. To illustrate, one text notes, "in the same way we have learnt to ask, when ordering a test, "How will the results of this test change my management of the patient's care?[...],' we can help trainees by asking, 'How will this test (or treatment) help achieve the patient's goals of care?", 18

Several texts presented a divergent definition, in which GOC was closely related to or synonymous with medical intervention decisions. For example, "A GOC plan is a medical order that clarifies limitations of medical treatment for a present condition." 19 In other divergent examples, the phrase GOC was equated with cardiopulmonary resuscitation status and orders, decisions about mechanical ventilation, or intensive care unit admission. Finally, several texts stated that GOC is ambiguous and lacks a clear definition: “'Goals of Care' may mean goals/ values of patients to some, to others it is the treatments that achieve the goals, or to some other it means both." 20

\section{Dimensions of GOC}

Within the healthcare literature, we identified three major themes that highlight the importance and the value of the GOC concept for patients with serious illness (Table 2). First, GOC is described as a mechanism to promote and protect patient autonomy and patient-centered care. Second, the concept of GOC can help avoid unwanted care: "Having a good understanding of patients' goals of care $(\mathrm{GoC})$ [sic] is a critical element in the shared decision-making process and important in avoiding unnecessary admissions to hospital, invasive

GOC goals of care 
Table 2 The Key Themes That Highlight the Importance and the Value of the "Goals of Care" Concept for Patients with Serious Illness

\begin{tabular}{ll}
\hline \hline Key theme & Exemplary quotes \\
\hline Promote patient autonomy & "Ethically, goals of care discussions help
\end{tabular}
and patient-centered care formulate advance care decisions that are congruent with patient values, ensure respect for patient autonomy [...]." "Treatment decisions should be based on patients' goals of care to provide an ethical, patient-centered framework for decision-making."

Avoid unwanted care and identify valued care

"Lack of consensus related to goals of care can leave residents, family, and staff feeling confused and can contribute to residents receiving care they do not want or missing out on care they value." "[...] competencies in goals of care communication deserve special attention. These value-based discussions elicit treatment preferences and may decrease unwanted medical interventions at the end of life."

Confer psychological support for patients and their families

"Using Goals of Care to guide decisionmaking about medical therapy in patients with advanced medical conditions is expected to enhance the quality of patient care by addressing the aspects of medical care and emotional support as part of a broader global care plan. This would likely alleviate the uncertainties of patients and families regarding decisions that involve limitations of medical care and may reduce refusals and delays in the process."

"In contrast to an approach centered on treatments, one centered on understanding patients' goals of care not only improves end-of-life care, but can also decrease the psychological burden on families of critically ill patients [...]."

procedures, suffering, and prolongation of the dying process." ${ }^{\prime 17}$ Finally, multiple texts acknowledged that application of the concept of GOC can confer psychological and emotional support to patients and their families who are facing complex, difficult decisions.

Many texts analyzed in this study provided examples of GOC to answer the question: What are the potential goals of care from which patients can choose? We identified two distinct frameworks used by authors to answer this question: dichotomous or inclusive. In texts that applied a dichotomous approach, a GOC is one primary goal that is selected from a continuum of goals anchored by life extension on one end, and comfort on the other, or as a dichotomous choice between these two extremes. To illustrate, one study asked participants to "assess their goals of care (on a Comfort Only-Survival Only continuum)."21 Similarly, another text described "the primary goal of care: either care focused on extending life or care focused on maximizing comfort."22

Several texts explicitly described concerns with the dichotomous framework: "While it is true that many physicians view these approaches as mutually exclusive, [...] We are concerned that framing goals of care in terms of singular priorities risks propagating a notion of care as either palliative or curative and may impede integration of these approaches to patient care." 23 Thus, the inclusive approach allows for a broader range of goals and the potential to aim for multiple goals simultaneously. Beyond life prolongation and comfort, the inclusive approach also includes the potential goals of disease prevention, achieving a good death, achieving specific life goals (e.g., attending a family event or strengthening relationships), improving or maintaining quality of life, providing support for family members or caregivers, living at home, maintaining independence, or removing interventions felt to be burdensome. One text stated, "There are many possible goals of medical care, from prevention, to cure, to prolongation of life, to achieving a good death. Multiple goals may apply simultaneously, with no one being more important than the others." ${ }^{24}$ Both the dichotomous and inclusive approaches typically referred to example GOC as discrete, categorical options instead of as an open-ended, unbounded concept.

Several key themes of GOC pertained to ownership of and responsibility for GOC, and the relationship between patients and clinicians. First, GOC are generally considered patientcentered and belong, primarily, to patients. Many texts used possessive nouns to demonstrate the patient ownership of GOC: "Incorporating the patient's goals of care into discussions" and "identifying patient goals of care." Second, although GOC are patient-centered, clinician interactions are described as central to the application of GOC in clinical practice. One text described four primary clinician roles: “(1) clarify and confirm a patient's goals, (2) discuss the clinical implications of those goals, (3) determine whether all relevant goals have been considered, and (4) set priorities among goals when necessary." ${ }^{25}$ In other texts, the clinician role is variously described as eliciting, articulating, supporting, understanding, communicating, interpreting, specifying, and documenting patients' GOC. Several authors also noted that clinicians may have their own judgments and biases about patients' GOC, which can contribute to conflict: "Clinicians might use these results to consider what their goals of care are, when and in what ways their goals are convergent or divergent with the goals of patients and families, whether or not aspects of divergence are problematic, and what they should discuss with patients and families in order to arrive at shared goals."26

GOC are also widely recognized as dynamic and subject to change over time. One author acknowledged that "As the patient's disease progresses, goals of care may evolve, with some objectives and preferences being more emphasized or some less so." 27 Several texts emphasized the key role clinicians play in helping patients navigate changes in GOC: "The ability to sit with the patient and hear his/her grief as losses accumulate, goals are modified, and hope redefined is integral to communication in nursing care at the end of life." 28 Finally, the concept of prioritization and trade-offs was central to the description of GOC: "When goals of care are multiple — which is commonly the case - there will be the possibility of conflict or tension between goals that require resolution or prioritizing., 29 


\section{DISCUSSION}

The phrase "goals of care" is pervasive in the language of both clinicians and researchers who seek to improve the care for patients with serious illness. However, the specific use and meaning of the phrase are ambiguous, limiting the impact and utility of this potentially valuable concept. In this systematic review, we synthesized the existing healthcare literature using qualitative discourse analysis to generate a consensus-based, operational definition of GOC for future application by clinicians, researchers, and health policymakers. Key themes from discourse analysis of the literature support the following operational definition of GOC: the overarching aims of medical care for a patient that are informed by patients' underlying values and priorities, established within the existing clinical context, and used to guide decisions about the use of or limitation on specific medical interventions.

The use of GOC within the healthcare literature is common and widespread. In a previous systematic review of texts published in the palliative oncology literature between 2004 and 2009, the phrase GOC was found in 55 texts. ${ }^{3}$ Our study expands on this search strategy to include the healthcare literature from all medical specialties. We identified a dramatic increase in the use of GOC, to approximately 60 texts per year with a major focus on GOC. Texts focusing on GOC are published in a wide variety of journals. Less than one-third of all included texts in this study were in palliative care journals, and other common journal types included internal medicine, oncology, and pulmonary and critical care medicine. The dramatic increase in use and reach of the phrase GOC within the healthcare literature supports the promise and appeal of this concept, and also highlights the need for precision and clarity.

For clinicians who care for patients with serious illness, our findings point to an important discrepancy between the clinical and healthcare literature uses of GOC. This discrepancy is important to patient care; imprecise language in the context of serious illness can create unwarranted confusion for patients and their families while navigating an already challenging and complex process within the healthcare system. ${ }^{6}$ In a prior study of progress notes of critically ill patients within the EHR, we found that clinicians use GOC extensively in their documentation to indicate poor prognosis, to describe conflict with families, or to provide rationale for limitations on specific medical interventions. ${ }^{2}$ Clinicians rarely documented patientor family-focused goals, priorities, or values while applying the phrase GOC. Similarly, in an observational study of family conferences in the intensive care unit for which the physician overtly declared an intention to discuss GOC with patients' family members, fewer than $4 \%$ of spoken words pertained to patient preferences or values, and only $12 \%$ of conferences included any conversation of patient values about autonomy, independence, emotional well-being, relationships, physical function, cognitive function, or spirituality. ${ }^{30}$ The clinical use of GOC may overlook patient values, yet the operational definition uncovered in this study is founded upon the elucidation and application of patient values and priorities within a given clinical context. The extent, the cause, and the effect of this discrepancy in GOC terminology between clinicians and contributors to the healthcare literature remain unknown. Future studies should test whether behavioral interventions that promote the patient-focused concept of GOC uncovered in this study ultimately improve communication among clinicians, patients, and their families.

In this study, we found that the use of the phrase GOC was related to but distinct from advance directives (ADs) and advance care planning (ACP). ACP is a "process that supports adults at any age or stage of health in understanding and sharing their personal values, life goals, and preferences regarding future medical care" ${ }^{\prime 31}$ (emphasis added); written ADs formalize these preferences via instructional statements that direct future medical care and may include living wills and designation of a healthcare proxy. However, ADs, given their focus on future states, may fail to support in-the-moment, complex clinical decisions. ${ }^{32-36}$ In contrast, our findings describe GOC as a concept intended to align real-time treatment decisions with patients' values and priorities in a current, specific clinical context. Together, the complimentary but distinct concepts of ACP and GOC support decision-making across the spectrum of health and disease.

For researchers who aim to design interventions that improve care for patients with serious illness, the development of standardized definitions of key terms and phrases is critical to foster clear and precise scientific communication. For example, the delivery of goal-concordant care has become a fundamental outcome measure for high-quality end-of-life care, ${ }^{37-40}$ and this outcome measure is dependent on a clear and operationalized definition of patients' GOC. Two prior systematic reviews have examined the healthcare literature to establish conceptual definitions for several fundamental palliative care terms such as "actively dying," "end of life," and "palliative care." ${ }^{41,42}$ Our study advances this work by defining the emerging phrase "goals of care."

This study has strengths and limitations. Discourse analysis is an ideal approach to study concepts that lack explicit definition, because it is designed to uncover latent themes related to the function, use, and meaning of language and text within its natural context. ${ }^{43,44}$ Through this analysis, we exposed tacit attributes of the concept of GOC that have not been previously described. Based on our clinical experiences and prior research findings about the phrase GOC, we focused our discourse analysis on texts related to patients with serious, lifelimiting, or life-threatening illnesses. Thus, our findings may not extend to other patient populations and may not reflect the variation and meaning of the phrase GOC in clinical contexts not included in this study. We limited this study to texts in which GOC was a major focus within the title or abstract. 
Therefore, our systematic review and description of the use of GOC within the healthcare literature does not include texts in which GOC is a less emphasized concept, introducing the potential for selection bias.

\section{CONCLUSIONS}

The phrase "goals of care" is increasingly common within the healthcare literature pertaining to patients with serious illness. Through a discourse analysis of the literature, we developed an operational, consensus definition and conceptual model of "goals of care." This model and definition can facilitate improved communication among clinicians, patients, and families, and provide a framework for researchers who aim to improve the delivery of care for patients with serious illness.

Acknowledgments: We acknowledge Margaret L. Schwarze, MD, MPP, for her review of a prior version of this manuscript.

Corresponding Author: Jacqueline M Kruser, MD, MS; Northwestern University Feinberg School of Medicine, 633 N. St. Clair Street, 20th Floor, Chicago, IL 60611, USA (e-mail: jacqueline. kruser@northwestern.edu).

\section{Compliance with Ethical Standards:}

Conflict of Interest: JMK received support for her time, in part, from NIH/NHLBI grant K23 HL146890. GJW received speaking fees from VitalTalk, a 501c3 nonprofit communication skills training organization. The remaining authors report no other sources of funding or conflicts of interest.

Publisher's Note: Springer Nature remains neutral with regard to jurisdictional claims in published maps and institutional affiliations.

\section{REFERENCES}

1. Stanek S. Goals of care: a concept clarification. J Adv Nurs 2017;73:1302-14.

2. Kruser JM, Benjamin BT, Gordon EJ, et al. Patient and Family Engagement During Treatment Decisions in an ICU: A Discourse Analysis of the Electronic Health Record. Crit Care Med 2019.

3. Hui D, Mori M, Parsons HA, et al. The lack of standard definitions in the supportive and palliative oncology literature. J Pain Symptom Manage 2012;43:582-92.

4. NIH State-of-the-Science Conference Statement on improving end-of-life care. NIH Consens State Sci Statements 2004;21:1-26.

5. Zanartu C, Matti-Orozco B. Comfort measures only: agreeing on a common definition through a survey. Am J Hosp Palliat Care 2013;30:359.

6. Kelemen AM, Groninger H. Ambiguity in End-of-Life Care TerminologyWhat Do We Mean by "Comfort Care?". JAMA Intern Med 2018;178:1442-3.

7. Chen EK, Riffin C, Reid MC, et al. Why is high-quality research on palliative care so hard to do? Barriers to improved research from a survey of palliative care researchers. J Palliat Med 2014;17:782-7.

8. Ferris FD, Bruera E, Cherny $\mathbf{N}$, et al. Palliative cancer care a decade later: accomplishments, the need, next steps - from the American Society of Clinical Oncology. J Clin Oncol 2009;27:3052-8.

9. Hodges BD, Kuper A, Reeves $\mathbf{S}$. Discourse analysis. BMJ 2008;337:a879.

10. Zimmermann C. Acceptance of dying: a discourse analysis of palliative care literature. Soc Sci Med 2012;75:217-24.

11. Green J, Thorogood N. Qualitative methods for health research. 4th edition. ed. Los Angeles: SAGE; 2018.

12. Guest G. Planning and Preparing the Analysis. Applied Thematic Analysis: SAGE Publications; 2012.
13. Bradley EH, Curry LA, Devers KJ. Qualitative data analysis for health services research: developing taxonomy, themes, and theory. Health Serv Res 2007;42:1758-72.

14. Tracy SJ. Interview planning and design: Sampling, recruiting, and questioning. Qualitative Research Methods: Collecting Evidence, Crafting Analysis, Communicating Impact. First ed: Blackwell Publishing Ltd; 2013.

15. Corbin J, Strauss A. Grounded Theory Research - Procedures, Canons and Evaluative Criteria. Qualitative Sociology 1990;19:418-27.

16. Fischer GS, Alpert HR, Stoeckle JD, Emanuel LL. Can goals of care be used to predict intervention preferences in an advance directive? Arch Intern Med 1997; 157:801-7.

17. Lefkowitz A, Henry B, Bottoms J, Myers J, Naimark DM. Comparison of Goals of Care Between Hemodialysis Patients and Their Health Care Providers: A Survey. Can J Kidney Health Dis 2016;3:2054358116678207.

18. Kaldjian LC. Teaching practical wisdom in medicine through clinical judgement, goals of care, and ethical reasoning. J Med Ethics 2010;36:558-62.

19. Thomas RL, Zubair MY, Hayes B, Ashby MA. Goals of care: a clinical framework for limitation of medical treatment. Medical Journal of Australia 2014;201:452-5.

20. Chelluri LP. Goals of Care: Role of Physicians in the ICU. Chest 2015; 148:e184

21. Douglas SL, Daly BJ, Lipson AR. Differences in Predictions for Survival and Expectations for Goals of Care between Physicians and Family Surrogate Decision Makers of Chronically Critically Ill Adults. Res Rev J Nurs Health Sci 2017;3:74-84.

22. Covinsky KE, Landefeld CS, Teno J, et al. Is economic hardship on the families of the seriously ill associated with patient and surrogate care preferences? SUPPORT Investigators. Arch Intern Med 1996;156:173741.

23. Allen MB, Jesus JE. The language of goals of care: framing preferences at the end of life. Chest 2012;141:1126.

24. Wiese KT. Caring at the end of life: goals of care, sudden illness, withholding and withdraing care, and the last hours of life. The Prairie Rose: Official Publication of the North Dakota Nurses Association 2001.

25. Haberle TH, Shinkunas LA, Erekson ZD, Kaldjian LC. Goals of care among hospitalized patients: a validation study. Am J Hosp Palliat Care 2011;28:335-41.

26. Bogardus ST, Jr., Bradley EH, Tinetti ME. A taxonomy for goal setting in the care of persons with dementia. J Gen Intern Med 1998;13:675-80.

27. Lanken PN, Terry PB, Delisser HM, et al. An official American Thoracic Society clinical policy statement: palliative care for patients with respiratory diseases and critical illnesses. Am J Respir Crit Care Med 2008;177:912-27.

28. Peereboom K, Coyle N. Facilitating Goals-of-Care Discussions for Patients With Life-Limiting Disease-Communication Strategies for Nurses. Journal of Hospice \& Palliative Nursing 2012;14:251-8.

29. Kaldjian LC, Curtis AE, Shinkunas LA, Cannon KT. Goals of care toward the end of life: a structured literature review. Am J Hosp Palliat Care 2008;25:501-11.

30. Scheunemann LP, Cunningham TV, Arnold RM, Buddadhumaruk $P$, White DB. How clinicians discuss critically ill patients' preferences and values with surrogates: an empirical analysis. Crit Care Med 2015;43:757-64

31. Sudore RL, Lum HD, You JJ, et al. Defining Advance Care Planning for Adults: A Consensus Definition From a Multidisciplinary Delphi Panel. J Pain Symptom Manage 2017;53:821-32.el.

32. Halpern J, Arnold RM. Affective forecasting: an unrecognized challenge in making serious health decisions. J Gen Intern Med 2008;23:1708-12.

33. Perkins HS. Controlling death: the false promise of advance directives. Ann Intern Med 2007;147:51-7.

34. Fagerlin A, Schneider CE. Enough. The failure of the living will. The Hastings Center report 2004;34:30-42.

35. Kirschner KL. When written advance directives are not enough. Clin Geriatr Med 2005;21:193-209, x.

36. Tulsky JA. Beyond advance directives: importance of communication skills at the end of life. Jama 2005;294:359-65.

37. Turnbull AE, Hartog CS. Goal-concordant care in the ICU: a conceptual framework for future research. Intensive Care Med 2017;43:1847-9.

38. Sanders JJ, Curtis JR, Tulsky JA. Achieving Goal-Concordant Care: A Conceptual Model and Approach to Measuring Serious Illness Communication and Its Impact. J Palliat Med 2018;21:S17-S27. 
39. Turnbull AE, Sahetya SK, Colantuoni E, Kweku J, Nikooie R, Curtis JR. Inter-rater agreement of intensivists evaluating the goal-concordance of preference-sensitive ICU interventions. J Pain Symptom Manage 2018.

40. Turnbull AE, Bosslet GT, Kross EK. Aligning use of intensive care with patient values in the USA: past, present, and future. Lancet Respir Med 2019.

41. Hui D, De La Cruz M, Mori M, et al. Concepts and definitions for "supportive care," "best supportive care," "palliative care," and "hospice care" in the published literature, dictionaries, and textbooks. Support Care Cancer 2013;21:659-85.
42. Hui D, Nooruddin Z, Didwaniya N, et al. Concepts and definitions for "actively dying," "end of life," "terminally ill," "terminal care," and "transition of care": a systematic review. J Pain Symptom Manage 2014;47:77-89.

43. McCloskey R. A guide to discourse analysis. Nurse Res 2008;16:24-44.

44. Ford-Sumner S. Genre analysis: a means of learning more about the language of health care. Nurse Res 2006;14:7-17.

Publisher's Note Springer Nature remains neutral with regard to jurisdictional claims in published maps and institutional affiliations. 